PROCEEDINGS OF THE

AMERICAN MATHEMATICAL SOCIETY

Volume 131, Number 10, Pages 3123-3128

S 0002-9939(02)06853-3

Article electronically published on December 30, 2002

\title{
DIVERGENT CESÀRO AND RIESZ MEANS OF JACOBI AND LAGUERRE EXPANSIONS
}

\author{
CHRISTOPHER MEANEY
}

(Communicated by Andreas Seeger)

\begin{abstract}
We show that for $\delta$ below certain critical indices there are functions whose Jacobi or Laguerre expansions have almost everywhere divergent Cesàro and Riesz means of order $\delta$.
\end{abstract}

\section{INTRODUCTION}

1.1. Orthogonal expansions. Suppose that $(X, \mu)$ is a positive measure space, $\left(\varphi_{n}\right)_{n=0}^{\infty}$ is an orthogonal subset of $L^{2}(X, \mu)$, and $h_{n}=\left\|\varphi_{n}\right\|_{2}^{2}$ for all $n \geq 0$. If $f$ is a function on $X$ for which all the products $f . \overline{\varphi_{n}}$ are $\mu$-integrable, then $f$ has an orthogonal expansion

$$
\sum_{n=0}^{\infty} c_{n}(f) h_{n}^{-1} \varphi_{n}(x)
$$

with coefficients

$$
c_{n}(f)=\int_{X} f(x) \overline{\varphi_{n}(x)} d \mu(x), \quad \forall n \geq 0
$$

1.2. Cesàro means. As described in Zygmund's book [16, pp. 76-77], the Cesàro means of order $\delta$ of the expansion (11) are defined by

$$
\sigma_{N}^{\delta} f(x)=\sum_{n=0}^{N} \frac{A_{N-n}^{\delta}}{A_{N}^{\delta}} c_{n}(f) h_{n}^{-1} \varphi_{n}(x)
$$

where $A_{n}^{\delta}=\left(\begin{array}{c}n+\delta \\ n\end{array}\right)$. Theorem 3.1.22 in [16] says that if the Cesàro means converge, then the terms of the series have controlled growth.

Lemma 1.1. Suppose that $\lim _{N \rightarrow \infty} \sigma_{N}^{\delta} f(x)$ exists for some $x \in X$ and $\delta>-1$. Then

$$
\left|c_{N}(f) h_{N}^{-1} \varphi_{N}(x)\right| \leq C_{\delta} N^{\delta} \max _{0 \leq n \leq N}\left|\sigma_{n}^{\delta} f(x)\right|, \quad \forall N \geq 0 .
$$

Received by the editors February 26, 2002 and, in revised form, April 29, 2002.

2000 Mathematics Subject Classification. Primary 42C05, 33C45, 42C10.

Key words and phrases. Jacobi polynomial, Laguerre function, Cesàro mean, Riesz mean, Cantor-Lebesgue Theorem, uniform boundedness. 
1.3. Riesz means. Hardy and Riesz [6] had proved a similar result for Riesz means. Recall that the Riesz means of order $\delta \geq 0$ are defined for each $r>0$ by

$$
S_{r}^{\delta} f(x)=\sum_{0 \leq n<r}\left(1-\frac{n}{r}\right)^{\delta} c_{n}(f) h_{n}^{-1} \varphi_{n}(x) .
$$

Theorem 21 of $\left[6\right.$ tells us how the convergence of $S_{r}^{\delta} f(x)$ controls the size of the partial sums $S_{r}^{0} f(x)$.

Lemma 1.2. Suppose that $f$ has an orthogonal expansion and for some $\delta>0$ and $x \in X$ its Riesz means $S_{r}^{\delta} f(x)$ converges to $c$ as $r \rightarrow \infty$. Then

$$
\left|S_{r}^{0} f(x)-c\right| \leq A_{\delta} r^{\delta} \sup _{0<t \leq r+1}\left|S_{t}^{\delta} f(x)\right| .
$$

Note that we can write

$$
c_{n}(f) h_{n}^{-1} \varphi_{n}(x)=\left(S_{n}^{0} f(x)-c\right)-\left(S_{n-1}^{0} f(x)-c\right)=\mathbf{O}\left(n^{\delta}\right)
$$

and obtain the same growth estimates as in Lemma 1.1

Gergen [5] wrote formulae relating the Riesz and Cesàro means of order $\delta \geq 0$, from which the equivalence of the two methods of summation follows.

1.4. Uniform boundedness. Suppose there is a $1<q \leq \infty$ for which $\varphi_{n} \in$ $L^{q}(X, \mu)$ for all $n$. In addition, suppose that there is some positive number $\lambda$ with

$$
\left\|\varphi_{n}\right\|_{q} \geq c n^{\lambda}, \quad \forall n \geq 1 .
$$

The formation of the coefficient $f \mapsto c_{n}(f)$ is then a bounded linear functional on the dual of $L^{q}(X, \mu)$ with norm bounded below by a constant multiple of $n^{\lambda}$. The uniform boundedness principle implies that for $p$ conjugate to $q$ and each $0 \leq \varepsilon<\lambda$ there is an $f \in L^{p}(X, \mu)$ so that

$$
c_{n}(f) / n^{\varepsilon} \rightarrow \infty \text { as } n \rightarrow \infty .
$$

1.5. Cantor-Lebesgue Theorem. The following argument is based on 16, Section IX.1]. Suppose we have a sequence of functions $F_{n}$ on an interval in the real line with the asymptotic property

$$
F_{n}(\theta)=c_{n}\left(\cos \left(M_{n} \theta+\gamma_{n}\right)+\mathbf{o}(1)\right), \quad \forall n \geq 0,
$$

uniformly on a set $E$ of finite positive measure, and with $M_{n} \rightarrow \infty$ as $n \rightarrow \infty$. Integrating $\left|F_{n}\right|^{2}$ over $E$ gives

$$
\begin{aligned}
& \int_{E}\left|F_{n}(\theta)\right|^{2} d \theta=\left|c_{n}\right|^{2}\left(\int_{E} \cos ^{2}\left(M_{n} \theta+\gamma_{n}\right) d \theta+\mathbf{o}(1)\right) \\
& =\left|c_{n}\right|^{2}\left(\frac{|E|}{2}+\frac{e^{2 i \gamma_{n}}}{4} \widehat{\chi_{E}}\left(2 M_{n}\right)+\frac{e^{-2 i \gamma_{n}}}{4} \widehat{\chi_{E}}\left(-2 M_{n}\right)+\mathbf{o}(1)\right) .
\end{aligned}
$$

The Riemann-Lebesgue Theorem [16, Thm. II.4.4] says that the Fourier transforms $\widehat{\chi_{E}}\left( \pm 2 M_{n}\right) \rightarrow 0$ as $M_{n} \rightarrow \infty$. If we know that there is some function $G$ for which $\left|F_{n}(\theta)\right| \leq G(n)$ uniformly on $E$ for all $n$, then there is an $n_{0}>0$ for which

$$
\frac{|E|}{4}\left|c_{n}\right|^{2} \leq \int_{E}\left|F_{n}(\theta)\right|^{2} d \theta \leq G(n)^{2}|E|, \quad \forall n \geq n_{0} .
$$

This shows that $\left|c_{n}\right| \leq 2 G(n)$ for all $n \geq n_{0}$. 


\section{JACOBI POLYNOMIALS}

2.1. Notation. Fix real numbers $\alpha \geq \beta \geq-1 / 2$, with $\alpha>-1 / 2$, and let $\mu$ denote the measure on $[-1,1]$ defined by

$$
d \mu(x)=(1-x)^{\alpha}(1+x)^{\beta} d x .
$$

Let $P_{n}^{(\alpha, \beta)}(x)$ be the Jacobi polynomial of degree $n$ associated to the pair $(\alpha, \beta)$ as in Szegö's book [15. Then $\left(P_{n}^{(\alpha, \beta)}\right)_{n=0}^{\infty}$ is an orthogonal subset of $L^{2}([-1,1], \mu)$. Equation (4.3.3) in [15] shows that the normalization terms $h_{n}^{(\alpha, \beta)}=\left\|P_{n}^{(\alpha, \beta)}\right\|_{2}^{2}$ satisfy

$$
h_{n}^{(\alpha, \beta)} \sim c_{\alpha, \beta} n^{-1} \text { as } n \rightarrow \infty .
$$

The Jacobi polynomial expansion of $f \in L^{1}(\mu)$ is

$$
\sum_{n=0}^{\infty} c_{n}(f)\left(h_{n}^{(\alpha, \beta)}\right)^{-1} P_{n}^{(\alpha, \beta)}(x),
$$

with coefficients $c_{n}(f)=\int_{-1}^{1} f(x) P_{n}^{(\alpha, \beta)}(x) d \mu(x)$. We take $\alpha$ and $\beta$ as fixed and use $\sigma_{N}^{\delta} f(x)$ and $S_{r}^{\delta} f(x)$ to denote the Cesàro and Riesz means of this expansion, respectively.

2.2. Asymptotics. Theorem 8.21 .8 in Szegö's book 15] gives the following asymptotic behaviour for the Jacobi polynomials.

Lemma 2.1. For $\alpha \geq \beta \geq-1 / 2$ and $\varepsilon>0$ the following estimate holds uniformly for all $\varepsilon \leq \theta \leq \pi-\varepsilon$ and $n \geq 1$ :

$$
P_{n}^{(\alpha, \beta)}(\cos \theta)=n^{-1 / 2} k(\theta) \cos \left(M_{n} \theta+\gamma\right)+\mathbf{O}\left(n^{-3 / 2}\right) .
$$

Here $k(\theta)=\pi^{-1 / 2}(\sin (\theta / 2))^{-\alpha-1 / 2}(\cos (\theta / 2))^{-\beta-1 / 2}, M_{n}=n+(\alpha+\beta+1) / 2$, and $\gamma=-(\alpha+1 / 2) \pi / 2$.

From Egoroff's theorem and Lemma 1.1 we can say that if $\sigma_{N}^{\delta} f(x)$ converges on a set of positive measure in $[-1,1]$, then there is a set of positive measure $E$ on which

$$
\left|c_{n} n^{(1 / 2)-\delta}\left(\cos \left(M_{n} \theta+\gamma\right)+\mathbf{O}\left(n^{-1}\right)\right)\right| \leq A
$$

uniformly for $\cos \theta \in E$. The argument of subsection 1.5 shows that

$$
\left|c_{n} n^{(1 / 2)-\delta}\right| \leq A, \quad \forall n \geq 1 .
$$

2.3. Norm estimates. Next we recall the calculation of Lebesgue norms of Jacobi polynomials, according to Markett [10] and Dreseler and Soardi [4]. Equation (2.2) in [10] gives the following lower bounds on these norms.

Lemma 2.2. For real numbers $\alpha \geq \beta \geq-1 / 2$, with $\alpha>-1 / 2,1 \leq q<\infty$, and $r>-1 / q$,

$$
\left(\int_{0}^{1}\left|P_{n}^{(\alpha, \beta)}(x)(1-x)^{r}\right|^{q} d x\right)^{1 / q} \sim \begin{cases}n^{-1 / 2} & \text { if } r>\alpha / 2+1 / 4-1 / q \\ n^{-1 / 2}(\log n)^{1 / q} & \text { if } r=\alpha / 2+1 / 4-1 / q \\ n^{\alpha-2 r-2 / q} & \text { if } r<\alpha / 2+1 / 4-1 / q\end{cases}
$$


2.4. Main result. There are critical indices, as used in [1],

$$
p_{c}=\frac{4(\alpha+1)}{(2 \alpha+3)} \text { and its conjugate } p_{c}^{\prime}=\frac{4(\alpha+1)}{(2 \alpha+1)} .
$$

Taking $r=\alpha / q$ in Lemma 2.2 we have that

$$
\left\|P_{n}^{(\alpha, \beta)}\right\|_{L^{q}(\mu)}>\left(\int_{0}^{1}\left|P_{n}^{(\alpha, \beta)}(x)\right|^{q}(1-x)^{\alpha} d x\right)^{1 / q} \sim n^{\alpha-2 \alpha / q-2 / q}
$$

for $\alpha / q<\alpha / 2+1 / 4-1 / q$. This last inequality can be rewritten as

$$
q>\frac{4(\alpha+1)}{2 \alpha+1}=p_{c}^{\prime} .
$$

We can now prove that below the critical index there are functions with almost everywhere divergent Cesàro and Riesz means.

Theorem 2.3. For real numbers $\alpha \geq \beta \geq-1 / 2$, with $\alpha>-1 / 2$,

$$
1 \leq p<p_{c}=\frac{4(\alpha+1)}{(2 \alpha+3)} \text {, and } 0 \leq \delta<\frac{(2 \alpha+2)}{p}-\frac{(2 \alpha+3)}{2},
$$

there is an $f \in L^{p}(\mu)$, supported in $[0,1]$, whose Cesàro means $\sigma_{N}^{\delta} f(x)$ and Riesz means $S_{r}^{\delta} f(x)$ are divergent almost everywhere on $[-1,1]$.

Proof. Let $q$ be conjugate to $p$, so that $1 / p=(q-1) / q$. Suppose that

$$
\delta<\frac{(2 \alpha+2)}{p}-\frac{(2 \alpha+3)}{2}=\frac{(2 \alpha+2)(q-1)}{q}-\frac{(2 \alpha+3)}{2}=\alpha+\frac{1}{2}-\frac{(2 \alpha+2)}{q} .
$$

Then

$$
\delta-\frac{1}{2}<\alpha-\frac{(2 \alpha+2)}{q}=\alpha-\frac{2 \alpha}{q}-\frac{2}{q},
$$

which is the exponent of $n$ in the inequality (10). Now apply the argument given in subsection 1.4 The norms of the Jacobi polynomials in Lemma 2.2 are calculated over $[0,1]$ and so we can find $f$ in $L^{p}([-1,1], \mu)$, supported on $[0,1]$, for which the coefficients satisfy

$$
c_{n}(f) / n^{\delta-1 / 2} \rightarrow \infty, \quad \text { as } n \rightarrow \infty .
$$

Combine this with Lemmas 1.1 and 1.2 and the argument around inequality (9) to see that for this $f$ both $\sigma_{N}^{\delta} f(x)$ and $S_{r}^{\delta} f(x)$ are divergent almost everywhere. This argument follows the methods used in [11, 8, 7 [12].

2.5. Remarks. Convergence results above the critical index are contained in the work of Bonami and Clerc [1, Colzani, Taibleson and Weiss [3], and Chanillo and Muckenhoupt 2]. In particular, in 2, Thm. 1.4] it is shown that for

$$
1 \leq p<p_{c}=\frac{4(\alpha+1)}{(2 \alpha+3)} \text { and } \delta=\frac{(2 \alpha+2)}{p}-\frac{(2 \alpha+3)}{2},
$$

the maximal operator $f \mapsto \sup _{N \geq 0}\left|\sigma_{N}^{\delta} f(x)\right|$ is of weak type $(p, p)$.

For $\delta=0$ and $p=p_{c}$, divergence was proved in [11]. 


\section{LAGUERRE FUnCTIONS}

3.1. Notation. For each $\alpha>-1$ let $\mu_{\alpha}$ be the measure on $[0, \infty)$ defined by

$$
d \mu_{\alpha}(x)=e^{-x} x^{\alpha} d x .
$$

We denote by $L_{n}^{(\alpha)}(x)$ the Laguerre polynomial of degree $n$, as in [15, Chpt. 5]. The $L^{2}\left(\mu_{\alpha}\right)$-norms of these satisfy the identity

$$
h_{n}^{(\alpha)}=\left\|L_{n}^{(\alpha)}\right\|_{L^{2}\left(\mu_{\alpha}\right)}^{2}=\Gamma(\alpha+1)\left(\begin{array}{c}
n+\alpha \\
n
\end{array}\right) \sim n^{\alpha} .
$$

Fejér's formula [15, Thm. 8.22.1] gives the asymptotic properties of these polynomials. For each $\alpha>-1$ and $0<\varepsilon<\omega$,

$$
L_{n}^{(\alpha)}(x)=\frac{e^{x / 2}}{\pi^{1 / 2} x^{\alpha / 2}} n^{\alpha / 2-1 / 4} \cos \left(2(n x)^{1 / 2}-\alpha \pi / 2-\pi / 4\right)+\mathbf{O}\left(n^{\alpha / 2-3 / 4}\right),
$$

uniformly in $x \in[\varepsilon, \omega]$. The corresponding normalized functions are

$$
\mathcal{L}_{n}^{\alpha}(x)=\sqrt{\frac{\Gamma(n+1)}{\Gamma(n+\alpha+1)}} e^{-x / 2} x^{a / 2} L_{n}^{(\alpha)}(x), \quad \forall x \geq 0, n \geq 0 .
$$

These provide an orthonormal subset of $L^{2}([0, \infty))$, where the half line carries Lebesgue measure.

3.2. Norm estimates. Markett [9, Lemma 1] has calculated the Lebesgue norms of the Laguerre functions, for $\alpha>-1 / 2$,

$$
\left\|\mathcal{L}_{n}^{\alpha}\right\|_{q} \sim \begin{cases}n^{1 / q-1 / 2}, & \forall 1 \leq q<4, \\ n^{-1 / 4}(\log n)^{1 / 4}, & \text { if } q=4, \\ n^{-1 / q}, & \forall 4<q \leq \infty .\end{cases}
$$

\subsection{Divergence result.}

Theorem 3.1. If $\alpha>-1 / 2, p>4$ and $0<\delta<1 / 4-1 / p$, then there is a function $f \in L^{p}(0, \infty)$ whose Laguerre expansion

$$
\sum_{n=0}^{\infty} c_{n}(f) \mathcal{L}_{n}^{\alpha}(x)
$$

has Cesàro and Riesz means of order $\delta$ which diverge almost everywhere.

Proof. Suppose that the expansion $\sum_{n=0}^{\infty} c_{n}(f) \mathcal{L}_{n}^{\alpha}(x)$ is either Cesàro or Riesz summable of order $\delta$ on a set of positive measure in $[0, \infty)$. Then Lemma 1.1 or Lemma 1.2 implies that

$$
c_{n}(f) \mathcal{L}_{n}^{\alpha}(x)=\mathbf{O}\left(n^{\delta}\right)
$$

on a set of positive measure. When equations (12) and (13) are combined with the argument of subsection 1.5 we find that

$$
c_{n}(f)=\mathbf{O}\left(n^{\delta+1 / 4}\right) .
$$

The case when $\delta=0$ is Lemma 2.3 in Stempak's paper [14]. Suppose that

$$
\frac{1}{q}-\frac{1}{2}>\delta+\frac{1}{4}
$$


so that $\delta<\frac{1}{q}-\frac{3}{4}=\frac{4-3 q}{q}$. If $\frac{1}{q}=1-\frac{1}{p}$, then this inequality is $\delta<\frac{1}{4}-\frac{1}{p}$. The argument of subsection 1.4 shows that if $p>4$ and $\delta<1 / 4-1 / p$, then there is a function $f \in L^{p}(0, \infty)$ for which the inequality (16) fails,

$$
c_{n}(f) / n^{\delta+1 / 4} \rightarrow \infty \text { as } n \rightarrow \infty \text {. }
$$

The Laguerre expansion of this function has Cesàro and Riesz means of order $\delta$ which diverge almost everywhere.

3.4. Remarks. There is an extensive treatment of almost everywhere convergence results for Laguerre expansions in [13. In particular, 13. Thm. 1.20] implies that if $p>4$ and $\delta \geq 1 / 4-1 / p$, then all $f \in L^{p}(0, \infty)$ have almost everywhere convergent Cesàro means of order $\delta$.

\section{REFERENCES}

1. Aline Bonami and Jean-Louis Clerc, Sommes de Cesàro et multiplicateurs des développements en harmoniques sphériques, Trans. Amer. Math. Soc. 183 (1973), 223-263. MR 49:3461

2. Sagun Chanillo and Benjamin Muckenhoupt, Weak type estimates for Cesàro sums of Jacobi polynomial series, Mem. Amer. Math. Soc. 102 (1993), no. 487, viii+90. MR 93g:42018

3. Leonardo Colzani, Mitchell H. Taibleson, and Guido Weiss, Maximal estimates for Cesàro and Riesz means on spheres, Indiana Univ. Math. J. 33 (1984), no. 6, 873-889. MR 86g:43012

4. B. Dreseler and P. M. Soardi, A Cohen-type inequality for Jacobi expansions and divergence of Fourier series on compact symmetric spaces, J. Approx. Theory 35 (1982), no. 3, 214-221. MR 84d:42029

5. J.J. Gergen, Summability of double Fourier series, Duke Math. J. 3 (1937), 133-148.

6. G. H. Hardy and M. Riesz, A general theory of Dirichlet series, Cambridge University Press, Cambridge, 1915.

7. Yūichi Kanjin, Convergence almost everywhere of Bochner-Riesz means for radial functions, Ann. Sci. Kanazawa Univ. 25 (1988), 11-15. MR 90b:42034

8. _ Convergence and divergence almost everywhere of spherical means for radial functions, Proc. Amer. Math. Soc. 103 (1988), no. 4, 1063-1069. MR 89i:42030

9. C. Markett, Mean Cesàro summability of Laguerre expansions and norm estimates with shifted parameter, Anal. Math. 8 (1982), no. 1, 19-37. MR 83j:40004

10. Cohen type inequalities for Jacobi, Laguerre and Hermite expansions, SIAM J. Math. Anal. 14 (1983), no. 4, 819-833. MR 85d:42025

11. Christopher Meaney, Divergent Jacobi polynomial series, Proc. Amer. Math. Soc. 87 (1983), no. 3, 459-462. MR 84c:42040

12. Christopher Meaney and Elena Prestini, Bochner-Riesz means on symmetric spaces, Tohoku Math. J. (2) 50 (1998), no. 4, 557-570. MR 99j:43003

13. Benjamin Muckenhoupt and David W. Webb, Two-weight norm inequalities for Cesàro means of Laguerre expansions, Trans. Amer. Math. Soc. 353 (2001), no. 3, 1119-1149. MR 2001m:42051

14. Krzysztof Stempak, Divergent Laguerre series, Proc. Amer. Math. Soc. 129 (2001), no. 4, 1123-1126. MR 2001g:42057

15. Gábor Szegő, Orthogonal polynomials, third ed., American Mathematical Society, Providence, R.I., 1967, American Mathematical Society Colloquium Publications, Vol. 23. MR 46:9631

16. A. Zygmund, Trigonometric series: Vols. I, II, Cambridge University Press, London, 1968. MR 38:4882

Department of Mathematics, Macquarie University, North Ryde, New South Wales 2109, Australia

E-mail address: chrism@maths.mq.edu.au 